\title{
MODIFICAÇ̃̃O DE TÉCNICA DE DETERMINAÇÃO DA CAPACIDADE DE TROCA CATIÔNICA DA FIBRA EM DETERGENTE NEUTRO DE ALIMENTOS
}

\author{
(Modification in the technique for cation-exchange capacity determination \\ in neutral detergent fiber of feeds)
}

\author{
WARPECHOWSKI, M.B. ${ }^{1 ;}$ SILVA, D.A. ${ }^{2}$
}

1'Departamento. de Zootecnia, Universidade Federal do Paraná; ${ }^{2}$ Médica Veterinária, ex-bolsista de iniciação científica UFPR-TN.

\begin{abstract}
RESUMO - A capacidade de troca catiônica (CTC) da fibra em detergente neutro (FDN) de amostras de fenos de azevém $(A Z)$ e de alfafa $(A L)$, de milho (Mi) e de farelos de soja (FS) e de trigo (FT) foi determinada através do método descrito por McBURNEY et al. (1983) e por método modificado, com seis repetições por material. A modificação consistiu em determinar a propriedade físico-química no resíduo da determinação de FDN ao invés da separação de grande quantidade de fibra para posterior avaliação da CTC. Os valores obtidos com o método modificado foram maiores que com o original, mas foi obtida a mesma classificação dos materiais estudados $(P<0,05)$, sendo que $\mathrm{FS}>\mathrm{Mi}>\mathrm{AZ}=\mathrm{AL}=\mathrm{FT}$. A correlação entre os métodos foi altamente significativa, com $r=0,9877(P<0,01)$. A alteração proposta economiza tempo e reagentes, e mostrou-se tão eficiente quanto o método original.
\end{abstract}

Palavras-chave: capacidade de troca catiônica; fibra em detergente neutro; método laboratorial; propriedades físico-químicas.

\begin{abstract}
The cation exchange capacity (CTC) of neutral detergent fiber (FDN) from samples of rye grass $(A z)$ and lucerne $(A L)$ hays, corn (Mi), soybean meal (FS) and wheat bran (FT) was evaluated by the original method developed by McBURNEY et al. (1983) and by modified technique of this method, with six replicates for each material and method. The modified method consisted in the determination of the physico-chemical property in the residue from FDN determination, instead of to separate a large quantity of fiber for subsequent CTC evaluation. It has been found that with the modified method higher values were found than the ones displayed by the original method, but the same classification has been observed among the studied materials $(P<0.05)$, with $\mathrm{FS}>\mathrm{Mi}>\mathrm{AZ}=\mathrm{AL}=\mathrm{FT}$. Correlation between methods was high, with $r=0.9877(P<0.01)$. On the other hand, the proposed modification saves time and reagents showing to be as efficient as the original method.
\end{abstract}

Key-words: cation exchange capacity; laboratory method; neutral detergent fiber; physico-chemical properties.

\section{Introdução}

Os efeitos fisiológicos e nutricionais da fibra dos alimentos, tanto para ruminantes quanto para monogástricos, são dependentes de sua composição e de suas propriedades físico-químicas (ANNISON e CHOCT, 1994; FERREIRA, 1994; VAN SOEST, 1994), as quais não podem ser quantificadas pela simples determinação da concentração de fibra. Dentre as propriedades físico-químicas da fibra insolúvel, a capacidade de troca catiônica se destaca por apresentar correlação com outras propriedades e efeitos nutricionais, além de permitir determinações repetíveis e de alta confiabilidade (MCBURNEY et al., 1983). Segundo a descrição de VAN SOEST et al. (1991) e ANNISON e CHOCT (1994) a CTC é a propriedade da fibra de ligar-se a íons metálicos através de grupos situados em sua superfície, o que estima a sua capacidade tamponante em uma determinada faixa de $\mathrm{pH}$. Segundo VAN SOEST (1994) a CTC da fibra da dieta tem sido apontada como um possível fator que afeta a disponibilidade de zinco, ferro e cobre para nãoruminantes. Além disso, essa medida estima a afinidade com cátions bivalentes utilizados como pontes para a fixação de microrganismos do trato digestório, influenciando na taxa e extensão da fermentação dos componentes da fibra (VAN SOEST et al., 1991). A capacidade tamponante influencia também na extensão e perfil da fermentação de alimentos ensilados (GIGERREVERDIN et al., 2002).

McBURNEY et al. (1983) desenvolveram método para a determinação da CTC da fibra em detergente neutro (FDN) de alimentos baseado na ligação da fibra com cátions de cobre em pH 3,5. O método é rápido e de relativo baixo custo, entretanto a determinação é feita em amostras da FDN do alimento, separada previamente por método adaptado para resultar em grande quantidade de resíduo fibroso. Essa separação prévia da fração fibrosa resulta em um período de no mínimo um dia a mais para a determinação da CTC, de equipamentos e procedimentos específicos, e no gasto de dois litros de solução de detergente neutro para cada 50-60 g de alimento, o que pode resultar na necessidade de grandes volumes de solução no caso de avaliação de alimentos com menor teor de fibra 
Modificação de técnica de determinação da capacidade de troca catiônica da fibra em...

(WARPECHOWSKI, 1996; WARPECHOWSKI e CIOCCA, 2005). Além disso, a separação em grande quantidade dificulta a filtração e lavagem do resíduo fibroso, incorrendo em risco de obtenção de resíduo com composição diferente do que seria obtido com o método original de determinação da FDN, como demonstram os valores de FDN menores que $90 \%$ obtidos anteriormente em resíduos de fibra de grãos de milho e de farelo de soja (WARPECHOWSKI e CIOCCA, 2005) e também de uma dieta completa para matrizes de frangos de corte (WARPECHOWSKI, 1996).

O objetivo do presente trabalho foi avaliar a possibilidade de determinação da capacidade de troca catiônica com cobre diretamente no resíduo proveniente da determinação de fibra em detergente neutro de diferentes alimentos para animais.

\section{Material e Métodos}

A caracterização dos materiais experimentais e 0 experimento de laboratório foram realizados nos Laboratórios de Nutrição Animal dos Departamentos de Zootecnia das Universidades Federais do Paraná e do Rio Grande do Sul, e as leituras em espectrofotômetro foram realizadas no Laboratório de Análises Clínicas do Hospital Veterinário da Universidade Federal do Paraná.

Os materiais experimentais utilizados foram fenos de azevém e de alfafa, o milho e os farelos de soja e de trigo, cuja composição bromatológica foi caracterizada para proteína bruta (PB), fibra bruta (FB), extrato etéreo (EE) e cinzas (CZ) conforme o AOAC (1975), e para fibra detergente neutro (FDN), fibra detergente ácido (FDA) e lignina insolúvel em ácido (LIG) conforme (GOERING e VAN SOEST, 1970), com no mínimo duas repetições para cada amostra em cada análise. A FDN das amostras que continham amido foi determinada utilizando-se 2,0 $\mathrm{mL}$ de alfa-amilase termoestável (TERMAMYL ${ }^{\circledR}$, Novo Nordisk do Brasil S.A.) por litro solução de detergente neutro (VAN SOEST et al., 1991).

A CTC da fibra em detergente neutro dos materiais experimentais foi determinada conforme a técnica descrita por McBURNEY et al. (1983) (M1) realizando a separação prévia de FDN na proporção de $50 \mathrm{~g} / 2 \mathrm{~L}$ de solução de detergente neutro com modificações descritas por WARPECHOWSKI e CIOCCA (2005), utilizando-se alfa-amilase termoestável nos materiais que continham amido; e pelo método alternativo (M2), na qual se determinou a CTC a partir do resíduo obtido na determinação de FDN, em duplicata adicional seca a $60^{\circ} \mathrm{C}$. Em cada corrida utilizou-se uma determinação de cada material em cada método. Para a avaliação individual dos métodos original (M1) e modificado (M2), utilizou-se arranjo fatorial $6 \times 5$ (seis corridas laboratoriais $X$ cinco materiais) e para a comparação entre os métodos utilizou-se arranjo fatorial $6 \times 5 \times 2$ (seis corridas laboratoriais $X$ cinco materiais $X$ dois métodos), totalizando 60 determinações.

O procedimento de separação da FDN levou de duas a três horas por material e as preparações de fibra foram secas a $60{ }^{\circ} \mathrm{C}$ em estufa de ar forçado por aproximadamente 24 horas. O resíduo foi moído em moinho tipo martelo com perfurações de $1 \mathrm{~mm}$, e alíquotas de 0,5000 $\mathrm{g}$ foram usadas na determinação da CTC pelo método M1.

No método M2, a separação prévia da fibra insolúvel foi realizada através do procedimento de determinação de FDN com as seguintes modificações: (a) a quantidade de amostra de cada material e o volume de solução de detergente neutro foram ajustados proporcionalmente de forma que o resíduo de fibra pesasse entre 0,3 e 0,6 g; (b) a tara e a pesagem dos cadinhos com fibra foi feita com precisão de $0,0001 \mathrm{~g}$ após secagem a $60^{\circ} \mathrm{C}$ em estufa de ar forçado por 12 horas; e (c) o resíduo de fibra seco nos cadinhos foi desagregado com auxílio de um bastão de vidro. Os cadinhos com fibra foram então utilizados na determinação da CTC sem mais modificações. Os valores de CTC dos dois métodos foram expressos em $\mathrm{mmol} \mathrm{H}^{+} / \mathrm{kg}$ de FDN. Os resultados dos métodos considerados individualmente foram submetidos a análise de variância e as médias comparadas pelo teste de Fisher - DMS com nível de significância de $5 \%$. Os resultados dos dois métodos tomados em conjunto foram submetidos à análise de variância e de correlação, com nível de significância de $5 \%$.

\section{Resultados e Discussão}

Os resultados da análise bromatológica dos materiais experimentais são apresentados na TABELA 1 e a caracterização da fibra insolúvel extraída dos materiais pelo método M1 pode ser observada na TABELA2. Os valores obtidos para matéria seca e cinzas estão dentro da variação encontrada na literatura (WARPECHOWSKI e CIOCCA, 2005).

TABELA 1 - COMPOSIÇÃO QUÍMICA PERCENTUAL DOS MATERIAIS EXPERIMENTAIS'.

\begin{tabular}{lcccccccc}
\hline & $\mathrm{MS}$ & $\mathrm{CZ}^{2}$ & $\mathrm{EE}^{2}$ & $\mathrm{~PB}^{2}$ & $\mathrm{FB}^{2}$ & $\mathrm{FDN}^{2}$ & $\mathrm{FDA}^{2}$ & $\mathrm{LIG}^{2}$ \\
\hline Milho & 97,2 & 1,4 & 5,4 & 9,0 & 2,2 & 13,7 & 3,4 & 0,48 \\
Far. de soja & 92,4 & 5,8 & 2,1 & 46,9 & 6,4 & 18,0 & 10,3 & 0,35 \\
Far. de trigo & 97,3 & 4,8 & 5,1 & 17,7 & 9,9 & 43,3 & 13,3 & 3,37 \\
Feno de azevém & 94,0 & 13,0 & 7,2 & 32,4 & 20,6 & 44,6 & 24,8 & 2,11 \\
Feno de alfafa & 91,9 & 7,6 & 3,6 & 19,6 & 28,5 & 49,2 & 37,2 & 5,79 \\
\hline
\end{tabular}

${ }^{1}$ Matéria seca $(\mathrm{MS})$, cinzas (CZ), extrato etéreo (EE), Proteína bruta (PB), fibra bruta (FB), fibra em detergente neutro (FDN), fibra em detergente ácido (FDA) e lignina (LIG). ${ }^{2}$ Expressos com base em $100 \%$ MS. 
Foi obtida alta recuperação de FDN na análise do resíduo de fibra separado dos alimentos pelo método M1, mesmo para milho e farelo de soja, o que difere do registrado por WARPECHOWSKI e CIOCCA (2005), os quais observaram valores de recuperação de FDN menores que $90 \%$ para estes materiais. Embora a metodologia tenha sido a mesma, a presente análise foi realizada em outro laboratório e com partidas diferentes de material. Por outro lado, verificou-se altos valores de proteína nos resíduos de fibra avaliados, e que foi maior nos materiais com alta proteína, e especialmente alto no resíduo proveniente do feno de azevém (TABELA 2). O feno de azevém utilizado continha alto teor de proteína, mas o alto resíduo de proteína na fibra do mesmo foi proporcionalmente maior se consideramos por exemplo o teor de proteína do farelo de soja e de sua fibra (TABELAS 1 e 2).

TABELA 2 - COMPOSIÇÃO QUÍMICA PERCENTUAL DO RESÍDUO DE FIBRA SEPARADO DOS MATERIAIS'.

\begin{tabular}{lcccc}
\hline & MS & Cinzas $^{2}$ & FDN $^{2}$ & PB $^{2}$ \\
\hline Milho & 94,61 & 0,89 & 94,00 & 6,12 \\
Far. Soja & 92,57 & 1,59 & 96,40 & 12,29 \\
Far. Trigo & 94,62 & 0,91 & 101,60 & 7,09 \\
Feno Azevém & 94,43 & 1,82 & 96,05 & 12,88 \\
Feno Alfafa & 94,13 & 2,00 & 98,16 & 9,98 \\
\hline
\end{tabular}

${ }^{1}$ Matéria seca (MS), fibra em detergente neutro (FDN), proteína bruta (PB). ${ }^{2}$ Expressos com base em $100 \%$ MS.

Os resultados da análise da CTC da fibra insolúvel dos materiais experimentais, determinados com o método original (McBURNEY et al, 1983) e com o método modificado, podem ser observados na TABELA 3.

TABELA 3 - VALORES DE CAPACIDADE DE TROCA CATIÔNICA (MMOL H+/KG FDN; MÉDIA \pm DESVIO PADRÃO) DA FIBRA INSOLÚVEL DE DIVERSOS MATERIAIS DETERMINADA PELO MÉTODO DE MCBURNEY et al. (1983) ORIGINAL (M1) E COM MODIFICAÇÕES (M2).

\begin{tabular}{lrlrlc}
\hline & \multicolumn{1}{c}{ M1 } & \multicolumn{1}{c}{ M2 } & Diferença $(\%$ M1) \\
\hline Farelo de soja & $203,3 \pm 43,8$ & $\mathrm{Ba}$ & $282,8 \pm 63,2$ & $\mathrm{Aa}$ & 39,1 \\
Milho & $148,5 \pm 59,9$ & $\mathrm{Bb}$ & $213,5 \pm 76,4$ & $\mathrm{Ab}$ & 43,8 \\
Feno de alfafa & $97,3 \pm 19,6$ & $\mathrm{Bc}$ & $128,9 \pm 28,2$ & $\mathrm{Ac}$ & 32,5 \\
Feno de azevém & $87,7 \pm 14,1$ & $\mathrm{Bc}$ & $96,2 \pm 22,4$ & $\mathrm{Ac}$ & 9,7 \\
Farelo de trigo & $67,1 \pm 9,9$ & $\mathrm{Bc}$ & $99,2 \pm 25,9$ & $\mathrm{Ac}$ & 47,8 \\
\hline Média & 120,8 & & 164,1 & 35,9 \\
\hline
\end{tabular}

Valores seguidos de letras distintas maiúsculas na linha e minúsculas na coluna diferem significativamente pela diferença mínima significativa de Fisher $(\mathrm{P}<0,01)$.

O método M1 apresentou maior precisão que M2, com erros padrão respectivamente de 11,66 e 16,51. Observa-se, pela TABELA 3, que com M2 obteve-se valores maiores que com M1 para todos os materiais estudados. Apesar das diferenças, os valores obtidos para ambos os métodos estão dentro do espectro de valores encontrados para estes alimentos na literatura (McBURNEY et al., 1983; VAN SOEST et al., 1991; WARPECHOWSKI e CIOCCA, 2005).

A fibra dos fenos de alfafa e de azevém apresentaram CTC menor que a da fibra de milho, o que não era esperado uma vez que, segundo McBURNEY et al. (1983), há em geral uma ordem crescente de CTC para farelos e grãos de cereais, feno de gramíneas e feno de alfafa. A alta CTC do FS já havia sido observada em outros trabalhos (McBURNEY et al., 1983; WARPECHOWSKI e CIOCCA, 2005).

A coincidência no agrupamento estatístico dos materiais estudados nos dois métodos observado na TABELA 3 demonstra congruência de $\mathrm{M} 2$ em relação a $\mathrm{M} 1$. $\mathrm{O}$ valor proporcionalmente menor obtido para o FA no M2 poderia ser explicado pela maior contaminação com proteína nos resíduo de fibra desse material utilizado no M1 (TABELA2).
McBURNEY et al. (1983) não comentam sobre um possível problema de contaminação ou processo de separação ineficiente da fibra e suas possíveis implicações nos valores determinados de CTC pelo método M1. Entretanto, aminas, carboxilas e outros grupos funcionais das proteínas são apontados como responsáveis por altos valores de CTC dessa fração química por diversos autores (McBURNEY et al., 1983; JERACI e VAN SOEST, 1990; ANNISON e CHOCT, 1994). Apesar disso, a análise de regressão entre os métodos demonstrou correlação alta e positiva $(r=0,9877$; $P<0,01$, FIGURA 1), confirmando equivalência nas duas formas de caracterização da CTC da fibra insolúvel de alimentos.

A utilização do método modificado permitiria a redução em pelo menos $24 \mathrm{~h}$ no período de análise e a economia de 1,4 a 1,8 litros de solução de detergente neutro por material analisado. Além disso, a metodologia M2 permite a determinação concomitante e complementar à determinação de rotina de FDN nos laboratórios, apresentando maior facilidade de adoção. Ainda assim os autores recomendam o aumento no espaço amostral na avaliação do método proposto, com a utilização de outros alimentos e de alimentos combinados e/ou dietas compostas. 
Modificação de técnica de determinação da capacidade de troca catiônica da fibra em...

FIGURA 1 - CORRELAÇÃO ENTRE OS MÉTODOS DE DETERMINAÇÃO DA CTC.

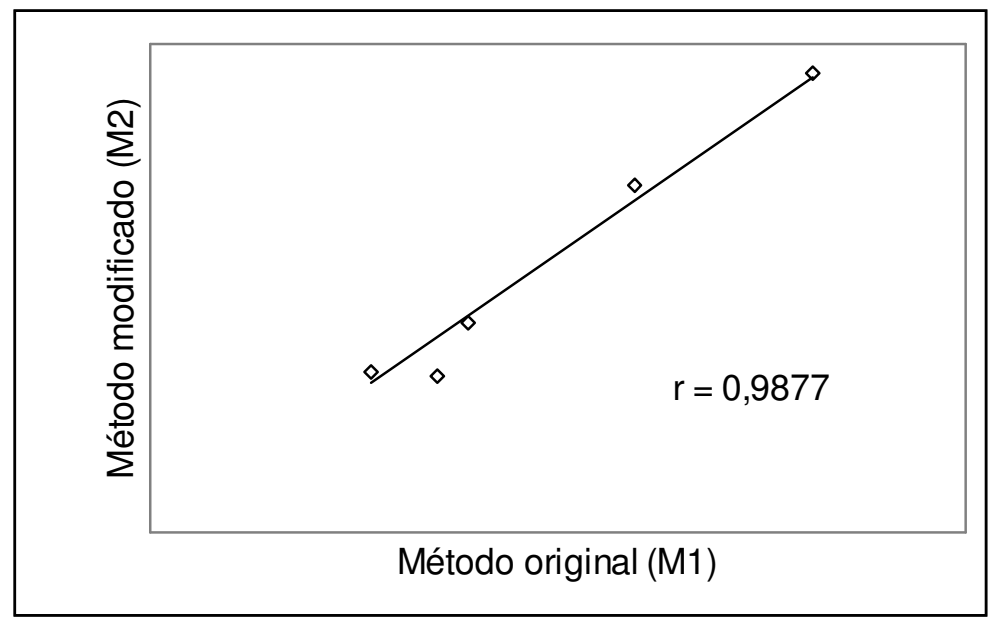

\section{Conclusões}

Conclui-se que a modificação proposta para o método de determinação da capacidade de troca catiônica da fibra insolúvel de alimentos resultou em medida mais fácil e de menor custo, sem prejuízo da eficiência de avaliação e classificação dos materiais estudados.

\section{Referências}

ANNISON, G.; CHOCT, M. Plant polysaccharides - their physiochemical properties and nutritional roles in monogastric animals. In: BIOTECHNOLOGY IN THE FEED INDUSTRY. PROCEDINGS OF ALLTECH'S TENTH ANNUAL SYMPOSIUM. 1994, Nottingham, Proccedings... Nottingham: Nottingham University Press, p.51-66. 1994.

ASSOCIATION OF OFFICIAL AGRICULTURAL CHEMISTS. Official Methods of Analysis. 12. ed., Washington. 1975. 1094p.

FERREIRA, W.M. Os componentes da parede celular vegetal na nutrição de não-ruminantes. Simpósio Internacional de Produção de Não-Ruminantes. In: Reunião Anual da Sociedade Brasileira de Zootecnia, XXXI. 1994, Maringá, Anais ... Maringá: SBZ. p.85-113. 1994.

GIGER-REVERDIN, S.; DUVAUX-PONTER, C.; SAUVANT, D.; MARTIN, O.; PRADO, I. N.; MÜLLER, R. Intrinsic buffering capacity of feedstuffs. Animal Feed Science and Technology, v.96, p.83-102, 2002.
GOERING, H. G.; VAN SOEST, P. J. Forage fiber analysis (apparatus, reagents, procedures and some aplications). Washington, Agriculture Research Service. 20p. (Agriculture Handbook, 379) 1970.

JERACI, J.L.; VAN SOEST, P.J. Improved methods for analysis and biological characterization of fiber. Advances in Experimental Medicine and Biology, v.270, p.245263. 1990.

McBURNEY, M. I.; VAN SOEST, P. J.; CHASE, L. E. Cation Exchange Capacity and Buffering Capacity of Neutraldetergent Fibres. Journal of the Science of Food and Agriculture, v.34, p.910-916, 1983.

VAN SOEST, P. J. Fiber and Physicochemical Properties of Feeds. In: Nutritional Ecology of the Ruminant. USA: Comstock Publishing Associates, 1994. p.140-155.

VAN SOEST, P.J.; ROBERTSON, J.B.; LEWIS, B.A. Methods for dietary fiber, neutral detergent fiber and nonstarch polysaccharides in relation to animal nutrition. Journal of Dairy Science, v.74, p.3583-3597. 1991.

WARPECHOWSKI, M. B.; CIOCCA, M. L. S. Propriedades físico-químicas da fibra em detergente neutro de alimentos isolados e misturados. Archives of Veterinary Science, v.11, n.1, p.23-26, 2006.

WARPECHOWSKI, M. B. Efeito da fibra insolúvel da dieta sobre a passagem no trato gastrintestinal de matrizes machos de linhagem de corte intactos, cecectomizados e fistulados no íleo terminal. Porto Alegre, RS. 117p. 1996. Dissertação (Mestrado em Zootecnia), Universidade Federal do Rio Grande do Sul, 1996.

Recebido para publicação: $\quad$ 15/12/2005 Aprovado: 